\title{
ORIGINAL RESEARCH \\ Subconjunctival Fat Prolapse and Dermolipoma of the Orbit: Differentiation on CT and MR Imaging
}

E. Kim

H.-J. Kim

Y.-D. Kim

K.I. Woo

H. Lee

S.T. Kim
BACKGROUND AND PURPOSE: Subconjunctival fat prolapse and dermolipoma of the orbit are unfamiliar to radiologists and yet should be differentiated because their treatments are different. The purpose of this study was to investigate the CT and MR imaging findings that distinguish these 2 conditions.

MATERIALS AND METHODS: Between 1995 and 2009, we found 33 patients (27 men, 6 women; mean age, 63 years) with surgically proved subconjunctival fat prolapse and 27 patients ( 3 males, 24 females mean age, 17 years) with dermolipoma in our hospital. Among them, 8 patients with subconjunctival fat prolapse and 6 patients with dermolipoma underwent CT and/or MR imaging examinations. We retrospectively reviewed $\mathrm{CT}$ scans and MR images in these patients.

RESULTS: In all of the 8 patients with subconjunctival fat prolapse, CT and MR images demonstrated the herniated fat at the superotemporal epibulbar area, continuous with the intraconal fat, extending forward between the lateral wall of the globe medially and the lateral rectus muscle and the lacrimal gland laterally, either bilaterally $(n=7)$ or unilaterally $(n=1)$. In contrast, all 6 patients with dermolipoma had a unilateral, crescent- $(n=5)$, or triangle-shaped $(n=1)$ fatty mass at the temporal or superotemporal epibulbar area, which lay on the lateral wall of the globe, anterior to the insertion of the lateral rectus muscle and medial to the lacrimal gland, without connection to the intraconal fat.

concLusıons: The characteristic CT and MR imaging findings may help easily differentiate subconjunctival fat prolapse and dermolipoma, both of which usually present as an epibulbar fatty mass in the lateral canthal area.
A lthough subconjunctival fat prolapse and dermolipoma are 2 distinct entities of the orbit that should be treated quite differently, both conditions look similar, usually presenting as a fat-containing epibulbar mass in the lateral canthal area and thus can confuse clinicians who are less experienced with them. ${ }^{1,2}$ Subconjunctival fat prolapse is an acquired lesion, characterized by a herniation of intraconal fat due to weakness of the Tenon capsule by the aging process, trauma, or surgery. ${ }^{1,3-6}$ In contrast, dermolipoma is a congenital choristomatous tumor containing dermis-like connective tissue and adipose tissue. ${ }^{7,8}$

Despite different pathogenesis and treatment, imaging studies for these lesions are rarely performed so that their imaging findings are unfamiliar to most radiologists. The purpose of this study was to investigate the CT and MR imaging findings that can distinguish subconjunctival fat prolapse and dermolipoma.

\section{Materials and Methods}

\section{Patients}

The institutional review board approved this retrospective study.

Between 1995 and 2009, a search of the electronic data base of our hospital revealed 33 patients (27 men, 6 women; age range, 43-81 years; mean, 63 years) with surgically proved subconjunctival orbital fat prolapse and 27 patients ( 3 males, 24 females; age range, 4-46 years; mean, 17 years) with dermolipoma. Among them, 8 patients with subconjunc-

Received June 12, 2010; accepted after revision August 13

From the Departments of Radiology (E.K., H.-J.K., H.L., S.T.K.) and Ophthalmology (Y.-D.K., K.I.W.), Samsung Medical Center, Sungkyunkwan University School of Medicine, Seoul, Korea.

Please address correspondence to Hyung-Jin Kim, MD, Department of Radiology, Samsung Medical Center, Sungkyunkwan University School of Medicine, 50 Ilwon-Dong, KangnamKu, Seoul 135-710, Korea; e-mail: hyungkim@skku.edu

DOI 10.3174/ajnr.A2313 tival fat prolapse and 6 patients with dermolipoma underwent CT or MR imaging examinations and formed the basis of this study.

All except 1 of 8 patients with subconjunctival fat prolapse presented with a fatty mass in the lateral canthal area either unilaterally $(n=1)$ or bilaterally $(n=6)$. The remaining patient presented with blurred vision in both eyes. All 6 patients with dermolipoma presented with a fatty mass in the lateral canthal area unilaterally. All patients with subconjunctival fat prolapse and dermolipoma denied a history of trauma or surgery of the eye.

\section{Imaging Techniques}

In the 8 patients with subconjunctival fat prolapse, CT scanning was performed in 4 patients and MR imaging, in 6 patients. Two patients underwent both CT and MR imaging examinations. In the 6 patients with dermolipoma, CT scanning was performed in 4 patients and MR imaging, in 2 patients. CT scans were obtained with and without contrast enhancement by using $2.5-$ to $3.0-\mathrm{mm}$ section thicknesses in both axial and coronal planes.

MR imaging examinations were performed on a $1.5 \mathrm{~T}(n=7)$ or 3T $(n=1)$ scanner to produce pre- and postcontrast T1-weighted spin-echo images and T2-weighted fast spin-echo images with or without fat saturation. Images were obtained in at least 2 planes with 3- to $5-\mathrm{mm}$ section thickness and 0.3 - to $1-\mathrm{mm}$ intersection gap.

\section{Image Analysis}

All CT scans and MR images were retrospectively reviewed by a dedicated head and neck neuroradiologist and a general neuroradiologist in conference, who have been practicing in the field for 20 years and 5 years, respectively. The imaging findings were assessed for the location, shape, size, and internal architecture (attenuation on CT scans and signal intensity on MR images) of the lesion. Because of continuation of the herniated fat with the intraconal fat in patients with subconjunctival fat prolapse, the lesion size was measured only in patients with dermolipoma by the maximum transverse and anteroposterior diameters on axial CT scans or MR images. The presence of 


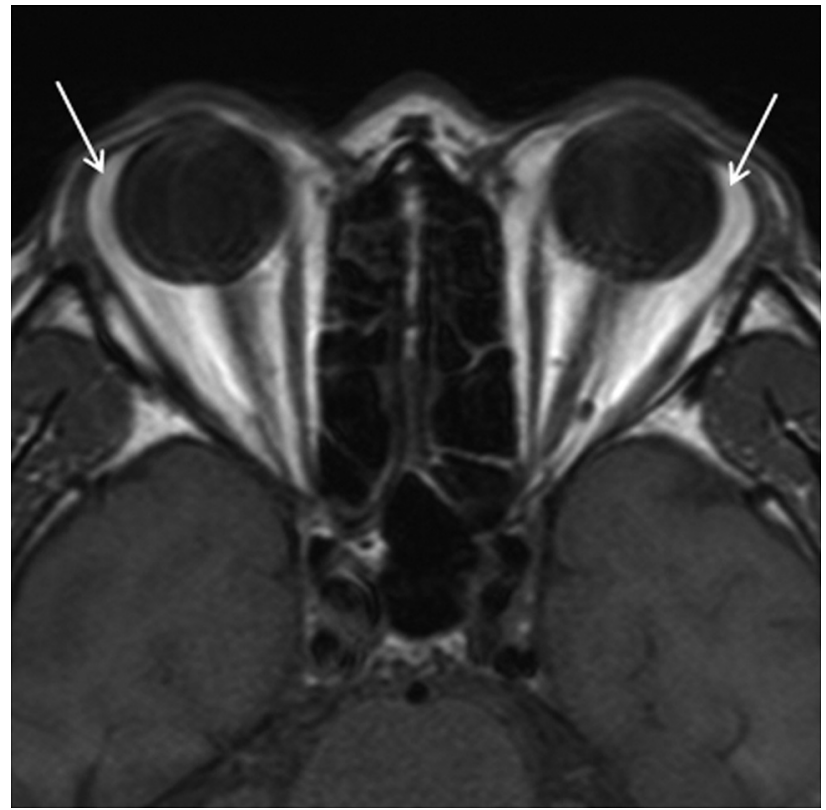

Fig 1. Bilateral subconjunctival fat prolapse in a 61-year-old man. Precontrast axial T1-weighted MR image demonstrates the herniated fat at the superotemporal aspect of bilateral epibulbar area, continuous with the intraconal fat, which extends anteriorly between the lateral wall of the globe medially and the lateral rectus muscle and the lacrimal gland laterally (arrows).

calcification determined on precontrast CT scans and the enhancement pattern, if any, were also recorded.

\section{Results}

In all 8 patients with subconjunctival fat prolapse, CT scans and MR images demonstrated the herniated fat at the superotemporal aspect of the epibulbar area, either bilaterally $(n=7$, Fig 1$)$ or unilaterally $(n=1$, Fig 2$)$. It was continuous with the intraconal fat, extending forward between the lateral wall of the globe medially and the lateral rectus muscle and the lacrimal gland laterally. In all lesions, the herniated fat was homogeneous. The attenuation on CT scans and the signal intensity on T1- and T2-weighted MR images of the lesions were identical to those of intraorbital fat, with no evidence of significant enhancement after injection of contrast material. In no case was intralesional calcification found on CT scans.

In all 6 patients with dermolipoma, CT scans and MR images showed a unilateral crescent- $(n=5)$ or triangle-shaped $(n=1)$ fatty mass at the temporal $(n=3)$ or superotemporal $(n=3)$ aspect of the epibulbar area, which lay on the lateral wall of the globe, anterior to the insertion of the lateral rectus muscle and medial to the lacrimal gland, without connection to the intraconal fat (Fig 3 ). The size of the lesions ranged from $8.7 \times 1.3 \mathrm{~mm}$ to $19.2 \times 6.6 \mathrm{~mm}$ (mean, $12.8 \times 3.9 \mathrm{~mm})$. While 4 lesions were seen as a homogeneous fatty mass whose attenuation on CT scans and signal intensity on MR images were identical to that of intraorbital fat, the remaining 2 lesions contained subtle soft-tissue strands within the mass on CT scans. There was no evidence of significant enhancement or calcification within the lesions.

\section{Discussion}

Both subconjunctival fat prolapse and dermolipoma usually occur in the lateral canthal area beneath the temporal or su-

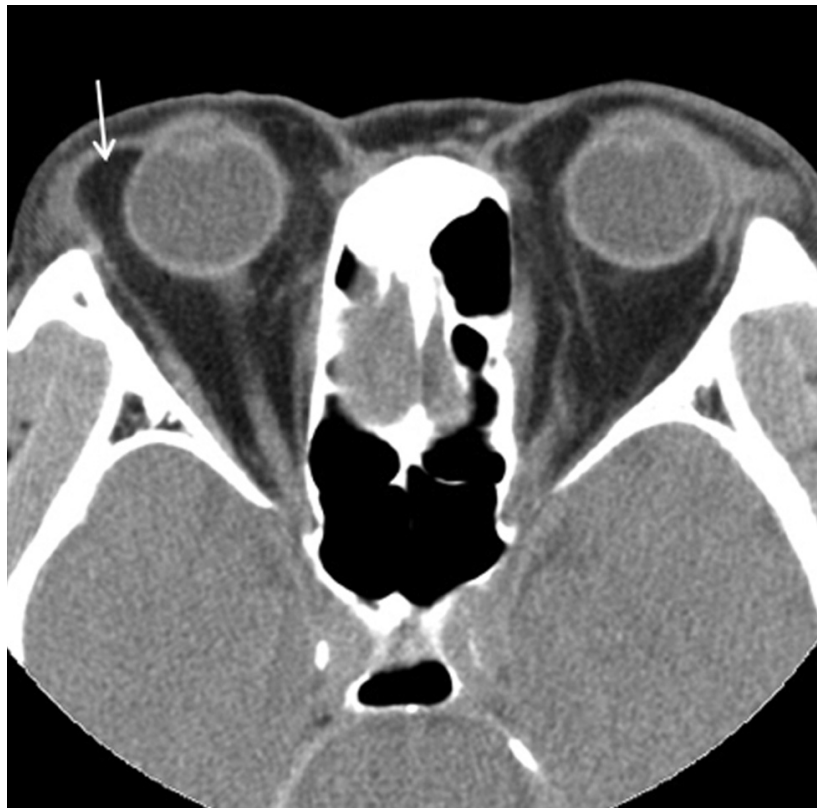

Fig 2. Unilateral subconjunctival fat prolapse in a 67-year-old man. Precontrast axial CT scan shows the herniated fat at the superotemporal aspect of the right epibulbar area (arrow). As in Fig 1, the prolapsed fat is continuous with the intraconal fat and lies between the lateral wall of the eyeball medially and the lateral rectus muscle and the lacrimal gland laterally.

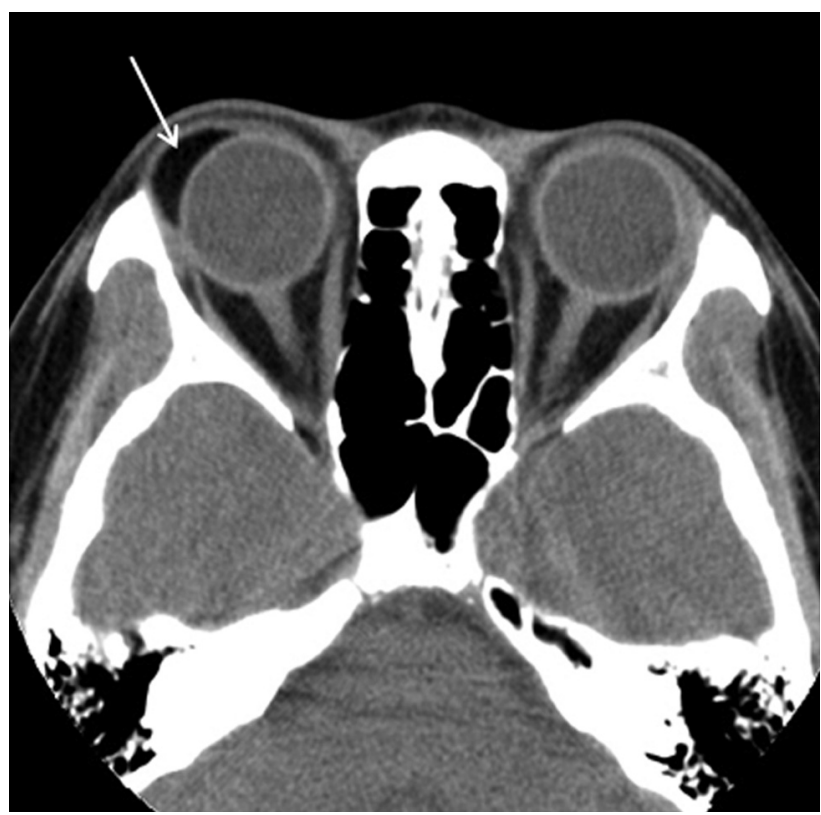

Fig 3. Dermolipoma in a 25-year-old woman. Precontrast axial CT scan demonstrates the crescent-shaped fatty mass at the superotemporal aspect of the right epibulbar area, which abuts the lateral wall of the eyeball, anterior to the insertion of the lateral rectus muscle and medial to the lacrimal gland (arrow). It has no connection to the intraconal fat. The lesion also contained a focal soft-tissue strand in the inferior portion (not shown).

perotemporal bulbar conjunctiva. Although the external appearance of these 2 conditions may be similar, certain demographic and clinical features are useful in distinguishing these conditions. Subconjunctival fat prolapse occurs mainly in elderly obese men with a mean age of 65-72 years (range, 28-94 years). ${ }^{6}$ It manifests typically as a unilateral or bilateral soft yellowish mass with a convex anterior margin and superficial fine blood vessels. It is easily repositioned into the orbit with a 
cotton tip applicator and becomes more prominent by retropulsion of the globe. ${ }^{1,2-4,6,9,10}$ Contrary to subconjunctival fat prolapse, dermolipoma is a congenital lesion occurring early in life with a female preference, with a mean age of 22.5 years (range, 1-68 years). ${ }^{7}$ It usually presents as a unilateral soft or firm pinkish-white or pinkish-yellow mass with a straight or slightly concave anterior margin, frequently accompanied by fine hairs on the surface. It is not so freely movable and cannot be repositioned into the orbit. The lesion size is not affected by retropulsion of the globe. ${ }^{1,2,4,7,8}$ Occasionally, the lesion may extend into the orbital fat and onto the bulbar conjunctiva, sometimes reaching the limbus. ${ }^{11}$

The pathogenesis of subconjunctival fat prolapse is believed to be a herniation of intraconal fat due to an acquired weakening of the Tenon capsule (fascia bulbi) and the intermuscular septum by the aging process. ${ }^{1,2,5,6,9}$ It can occasionally affect younger patients in association with trauma or surgery. ${ }^{10}$ Surgical removal through a perilimbal conjunctival incision can easily be done if the lesion is cosmetically unacceptable or causes discomfort. ${ }^{1,10}$

Dermolipoma is believed to be a congenital solid choristoma that is derived from an ectopia of the ectoderm to the conjunctiva, probably due to sequestration at the time of embryonic development of the eyelid. ${ }^{8}$ Although most lesions occur sporadically, the lesion may be associated with a congential anomaly such as Goldenhar syndrome, especially when there is a positive family history. ${ }^{7}$ Histologically, dermolipoma is characterized by the stratified squamous epithelium and the subepithelial stroma, which contains various amounts of collagenous connective tissue and a disproportionate amount of adipose tissue. The stroma may also contain cartilage and glandular acini. ${ }^{4,11,12}$ Surgical resection of dermolipoma should be done more conservatively, with limited debulking of the tumor. ${ }^{8}$ Attempts at a wide or complete surgical excision may result in complications such as ptosis, diplopia, and lacrimal gland injury, which are unacceptable for an essentially cosmetic problem. ${ }^{1,7}$

In the present study, the CT and MR imaging findings of subconjunctival fat prolapse and dermolipoma were quite different. The most important imaging feature distinguishing these 2 conditions was the fatty mass in question being continuous with the intraconal fat. Although both lesions were located at the temporal or superotemporal aspect of the epibulbar region in all cases, they were invariably continuous with the intraconal fat in cases of subconjunctival fat prolapse, while invariably isolated from the intraconal fat in cases of dermolipoma. In their study on the CT features of dermolipoma, Eijpe et $\mathrm{al}^{8}$ reported that the lesion extended posteriorly and appeared to be continuous with the orbital fat. However, other studies as well as the present one support the isolated feature of dermolipoma. ${ }^{1,13}$ On the basis of embryogenesis (ie, an ectopia of the ectoderm to the conjunctiva), it may be more usual to see dermolipoma in isolation from the orbital fat. In our study, CT scans showed subtle soft-tissue strands within 2 of 6 dermolipomas. This finding may represent the histologic feature of collagenous connective tissue contained within the stroma. Although there was a report of dermolipoma containing bone, ${ }^{13}$ our series did not show any significant calcific bodies within the lesion on CT scans.

Clinically, subconjunctival fat prolapse and dermolipoma may be difficult to distinguish from other pathologies of the conjunctiva and eyelids, such as dermoids, prolapsed lacrimal gland or lacrimal gland neoplasms, lymphomas, and lipomatous tumors. ${ }^{6}$ In those situations, imaging studies such as CT and MR imaging examinations may help narrow the differential diagnosis by demonstrating the fat component within the lesion, though histopathologic confirmation may ultimately be required to make a diagnosis. Radiologically, fat-containing epibulbar masses that should be included in the differential diagnosis are limbal dermoid and lipoma. Limbal dermoid is another important epibulbar chilostomatous lesion and is usually seen as a well-circumscribed firm solitary mass. ${ }^{11}$ Most are located inferotemporally but may arise also in the nasal or superior portions. ${ }^{12}$ Histologically, limbal dermoid is a simple choristoma, consisting of attenuated fibrous tissue lined by conjunctival epithelium with deeper dermal elements containing hair follicles and sebaceous glands. ${ }^{11}$

Except for the different predilection sites of occurrence, it may be difficult to differentiate dermolipoma and limbal dermoid radiologically. Sometimes cystic dermoid containing pilosebaceous and glandular structures in the wall may arise from the conjunctiva and extend posteriorly into the orbit. This lesion has been reported to have a propensity to arise in the superomedial aspect of the conjunctiva. ${ }^{12}$ Rarely, lipomatous tumors originating from the bulbar conjunctiva can mimic subconjunctival fat prolapse. ${ }^{6}$ Conjunctival lipoma often occurs bilaterally $(50 \%)$ and is mainly located in the upper fornix of the eye. ${ }^{6,14}$ Radiologically, conjunctival lipoma can be differentiated easily from subconjunctival fat prolapse by a well-circumscribed fatty mass that is isolated from the intraconal fat.

In summary, both subconjunctival fat prolapse and dermolipoma appear as an epibulbar fatty mass located in the lateral canthal area. Although certain demographic and clinical features are useful in distinguishing these 2 conditions, the characteristic CT and MR imaging findings may help make a correct diagnosis easily when the clinical diagnosis is in doubt.

\section{References}

1. Kim YD, Goldberg RA. Orbital fat prolapse and dermolipoma: two distinct entities. Korean J Ophthalmol 1994;8:42-43

2. Otaka I, Kyu N. A new surgical technique for the management of orbital fat prolapse. Am J Ophthalmol 2001;131:267-69

3. Glover AT, Grove AS. Subconjunctival orbital fat prolapse. Ophthal Plast Reconstr Surg 1987;3:83-86

4. Jordan DR, Tse DT. Herniated orbital fat. Can J Ophthalmol 1987;22:173-77

5. Monner J, Benito JR, Zayuelas J, et al. Transconjunctival herniation of orbital fat. Ann Plast Surg 1998;41:658-61

6. Schmack I, Patel RM, Folpe AL, et al. Subconjunctival herniated orbital fat: a benign adipocytic lesion that may mimic pleomorphic lipoma and atypical lipomatous tumor. Am J Surg Pathol 2007;31:193-98

7. McNab AA, Wright JE, Caswell AG. Clinical features and surgical management of dermolipomas. Aust N Z J Ophthalmol 1990;18:159-62

8. Eijpe AA, Koornneef L, Bras J, et al. Dermolipoma: characteristic CT appearance. Doc Ophthalmol 1990;74:321-28

9. Sato K, Yamaguchi T, Yokota H. A surgical technique with connective tissue repair for the management of subconjunctival orbital fat prolapse. Clin Experiment Ophthalmol 2006;34:841-45

10. McNab AA. Subconjunctival fat prolapse. Aust $N Z J$ Ophthalmol $1999 ; 27: 33-36$

11. Shields CL, Shields JA. Conjunctival tumors in children. Curr Opin Ophthalmol 2007;18:351-60

12. Spencer WH. Ophthalmic Pathology: An Atlas and Textbook. 4th ed. Philadelphia: Saunders; 1996:48-56

13. Lissner GS, Bryar P. Bone located centrally within a dermolipoma. Arch Ophthalmol 2003;121:730-32

14. Daniel CS, Beaconsfield M, Rose GE, et al. Pleomorphic lipoma of the orbit: a case series and review of literature. Ophthalmology 2003;110:101-05 